\title{
Free vibrations of nonlocally elastic rods
}

\section{G Mikhasev $^{1}$, E Avdeichik ${ }^{1}$ and D Prikazchikov ${ }^{2}$}

\begin{abstract}
Several of the Eringen's nonlocal stress models, including two-phase and purely nonlocal integral models, along with the simplified differential model, are studied in case of free longitudinal vibrations of a nanorod, for various types of boundary conditions. Assuming the exponential attenuation kernel in the nonlocal integral models, the integro-differential equation corresponding to the two-phase nonlocal model is reduced to a fourth order differential equation with additional boundary conditions taking into account nonlocal effects in the neighbourhood of the rod ends. Exact analytical and asymptotic solutions of boundary-value problems are constructed. Formulas for natural frequencies and associated modes found in the framework of the purely nonlocal model and its "equivalent" differential analogue are also compared. A detailed analysis of solutions suggests that the purely nonlocal and differential models lead to ill-posed problems.
\end{abstract}

\section{Keywords}

Eringen's nonlocal elasticity, two-phase integral model, nanorod, free longitudinal vibrations, asymptotic method, natural frequencies

\section{Introduction}

The first ideas of nonlocal theory of elasticity arise from the publications of Kröner [1], Kunin [2], Krumhansl [3], and Edelen and Laws [4], [5]. The conventional formulation of the nonlocal elasticity was completed by Eringen in [6], [7], and, [8], summarized in his well-known monograph [9]. After

\footnotetext{
${ }^{1}$ Department of Bio-and Nanomechanics, Belarusian State University,

4 Nezavisimosti Ave., 220030 Minsk, Belarus

${ }^{2}$ School of Computing and Mathematics, Keele University,

Keele, Staffordshire, ST5 5BG, UK

\section{Corresponding author:}

Gennadi Mikhasev, Department of Bio-and Nanomechanics, Belarusian State University,

4 Nezavisimosti Ave., 220030 Minsk, Belarus

Email: mikhasev@bsu.by
} 
these papers of Eringen, numerous contributions followed, studying deformations of nanoscale beams, plates and shells, as well as discrete nanostructures including nanotubes and graphene within the general framework of continuum mechanics.

According to the classical nonlocal elasticity, the stresses at a given local point of a continuum medium depend on the strains evaluated not only at the point itself, but also in the overall volume. Clearly, this general integral constitutive relation leads to sophisticated integro-differential equations, which are challenging even for 1D problems. Therefore, a simplified differential form was proposed by Eringen [6], which may be considered as equivalent to general integral formulation for certain problems, e.g. propagation of plane waves in an unbounded medium.

Over the last fifteen years, this differential formulation has been often employed as a framework for problems in stability and vibrations of nano- beams, plates and shells, as well as carbon nanotubes, see e.g. relatively early works [10,11], and also some more recent [12]-[18] to name a few. Below we refer to the model with general integral constitutive relation as "purely nonlocal integral model" (PNIM), and to the "effective" differential model as "differential nonlocal model" (DNM).

Adoption of this model led to a number of novel mechanical effects. These are mostly related to increase in deflections and decrease of the eigenfrequencies and critical loads as the effect of nonlocality. The only exception from this pattern seemed to be the behaviour of a cantilever nanobeam. Indeed, for the latter the action of a concentrated load does not reveal any nonlocal effects. This phenomena was noted in [10], and also in [19] and [20], implying that the DNM is not consistent. Moreover, it has been shown recently in [21], that the results provided by DNM for extension of a nanorod contradict the integral constitutive relations of PNIM based on the exponential kernel.

This contradiction is seemingly due to the formulation of certain boundary conditions and the choice of the kernel in the original PNIM as well. For example, the free ends boundary conditions within DNM are commonly written in terms of macroscopic stresses, see e.g. [12], [13], [16], which is in obvious violation with the general integral representation taking in consideration all of the near-boundary effects. So, for the case of fixed-free boundary conditions, Aydogdu [16] found the natural frequencies (see Eq. (15) in the paper) from the trigonometric solution which satisfies the boundary condition for a free end in terms of the macroscopic axial stress but not the nonlocal stress. As will be shown later, in case of kinematic boundary conditions (both ends are fixed) the differential model equivalent to PNIM with the exponential kernel does not account for boundary effects, caused by nonlocal effects of nano-structures.

Thus, in general case, DNM is inconsistent since it does not allow rigorous treatment of boundary conditions in terms of stresses, and does not provide an adequate description of deformations of a nanostructure in the near-boundary domain. Similar conclusions were presented in a recent work [22] for the conventional Gaussian kernel, comparing corrections arising from the boundary layer and the nonlocal effect within the media. It is concluded in the abovementioned paper, that the nonlocal effects within the media are of higher asymptotic order than those arising from rigorous asymptotic treatment of boundary conditions. The approach was later extended to thin Kirchhoff plates, for more detail see [23].

We also note interesting results obtained in [24] for static deflection of a nonlocally elastic beam subjected to transverse load of arbitrary profile. It turned out that the solutions provided by DNM and PNIM for the same load are in good agreement only when the loading satisfies additional conditions, formulated in [25].

Another situation, when DNM is apparently valid, is related to the case of strong localization of the field, occurring far away from the boundaries of a nano-structure. For example, we mention [26, 27], 
studying free localized vibrations of carbon nanotubes, incorporated into inhomogeneous elastic matrix. Indeed, within the framework of DNM applied to elongated thin shells the strongly localised solutions were obtained, decaying rapidly away from certain contours located far away from the ends of singlewalled and double-walled nanotubes, see [26] and [27], respectively.

As for pure nonlocal integral model (PNIM), its application to a problem of static bending of a beam [24] leads to a couple of canonical integral Fredholm equations of the first kind, hence, in case of arbitrary load, the problem is ill-posed, see e.g. [28]. A rigorous proof of the fact for bending of a beam relying on PNIM has recently appeared in [29].

The issue may be resolved, if one employs the so-called two-phase nonlocal model (TPNM), suggested by Eringen [30, 31], which takes into account contributions of both local and nonlocal components in the constitutive relation. We mention here the paper [20], along with more recent contributions [32], [33] and [34], applying TPNM to Euler-Bernoulli beams and roads. It has been shown in [29] that the problem of a static bending of a beam is well-posed and possesses a unique solution for TPNM. We also cite a recent treatment of Timoshenko beams within TPNM [35].

The two-phase model was also applied to other problems, for example, to extension of nanorods, see [21, 36]. The eigenforms for the Euler-Bernoulli beam were studied [37] using the discretisation of TPNM. At the same time, the authors are unaware of existing analytical methods for dynamics of nanobeams, plates and shells within the framework of TPNM. Such problems reduce to the Fredholm type integro-differential equations, differing significantly from the previously known static results. Therefore, additional investigation is of interest.

The aim of the present work is to study the problem of free longitudinal vibrations of a nanorod described within the TPNM framework. Since the two-phase model admits PNIM as a limiting case, as well as the conventional local elasticity theory, a comparison of natural frequencies will be performed, including those obtained for DNM.

The main body of this paper begins, in Section 2, from a brief description of the models of nonlocal elasticity, including PNIM, DNM and TPNM. Then, in Section 3 the formulation of the problem of free vibrations of a nanorod is presented. Then, some preliminary insights related to comparison of the nonlocal models, are discussed in Section 4. The exact and asymptotic solutions of the problem are derived in Section 5, leading to discussions and qualitative conclusions in Sections 6 and 7.

\section{Eringen's nonlocal models}

\subsection{Purely nonlocal integral model}

According to the purely nonlocal integral model of elasticity (PNIM), see e.g. [9], the stress tensor $\sigma_{k l}$ at any body point $\mathbf{x}=\left(x_{1}, x_{2}, x_{3}\right)$ is governed by the following constitutive relation

$$
\sigma_{i j}(\mathbf{x})=\int_{V} K\left(\left|\mathbf{x}-\mathbf{x}^{\prime}\right|, \varepsilon\right) \sigma_{i j}^{(c)}\left(\mathbf{x}^{\prime}\right) d V\left(\mathbf{x}^{\prime}\right),
$$

where $\sigma_{i j}^{(c)}$ is the macroscopic (classical) stress tensor at $\mathbf{x}^{\prime}, V$ is the region occupied by an elastic body, $K\left(\left|\mathbf{x}-\mathbf{x}^{\prime}\right|, \varepsilon\right)$ is the kernel or attenuation function which is positive and decays rapidly at $\left|\mathbf{x}-\mathbf{x}^{\prime}\right| \rightarrow \infty$, and $\varepsilon$ is the dimensionless nonlocal parameter reflecting the size effect and expressed as

$$
\varepsilon=\frac{e_{0} a}{l_{c}} \text {. }
$$


Here, $e_{0}$ is an appropriate material constant, $a$ is an internal characteristic length (e.g., lattice parameter, granular distance or $\mathrm{C}-\mathrm{C}$ bond), and $l_{c}$ is an external typical length (e.g., wave-length, a characteristic size of a body). In addition, when $\varepsilon \rightarrow 0$, the kernel function $K$ reverts to the Dirac delta function $\delta\left(\left|\mathbf{x}-\mathbf{x}^{\prime}\right|\right)$.

The choice of the kernel $K$ depends on the material and problem under consideration, its form and parameters being determined by matching the dispersion curves of plane waves with those of atomic lattice dynamics or experiments [7, 38]. The most commonly used kernel functions are exponential [8, 24, 33, 35, 36] and Gaussian [39, 40, 41] ones, sometimes the modified Bessel function is applied [8, 38, 42]. The exponential kernel are utilized, as a rule, in 1D-problems.

\subsection{Differential nonlocal model}

The differential nonlocal model (DNM) may be reduced from PNIM as follows [8]. We assume that $K$ is a Green's function of a linear differential operator $L_{E}$, i.e.

$$
L_{E} K\left(\left|\mathbf{x}-\mathbf{x}^{\prime}\right|, \tau\right)=\delta\left(\left|\mathbf{x}-\mathbf{x}^{\prime}\right|\right) .
$$

Then, on applying this operator to Eq. (1), one can obtain the following equation:

$$
L_{E} \sigma_{i j}(\mathbf{x})=\sigma_{i j}^{(c)}(\mathbf{x}) .
$$

In particular, considering the kernel $K$ in the form of 2D Gaussian function, Eringen [8] proved that $L_{E}$ is the diffusion operator, and for the kernel in the form of 2D Bessel function, he found

$$
L_{E}=\left(1-\varepsilon^{2} l_{c}^{2} \nabla^{2}\right),
$$

therefore,

$$
\left(1-\varepsilon^{2} l_{c}^{2} \nabla^{2}\right) \sigma_{i j}(\mathbf{x})=\sigma_{i j}^{(c)}(\mathbf{x}) .
$$

Eringen [8] has justified this equation by considering the Born-Kármán model of the crystal lattice dynamics and equating the derived expression of the frequency to that of plane waves obtained in the framework of the nonlocal elasticity theory. Also, matching the results obtained by different approaches, he found $e_{0} \simeq 0.39$. Recently published papers, which adopted this model (DNM) for studying vibrations of nonlocal beams, plates and shells, showed that the magnitude of $e_{0}$ is influenced by the type of vibration modes and scattered at the width range of zero to more than ten (e.g., see brief reviews of related papers in $[43,44])$. Furthermore, its application to carbon nanotubes has revealed that $e_{0}$ depends on the tube size and chirality as well [43].

We remind again that in spite of the inconsistency of DNM in a number of cases, it has been used in numerous studies for analysis of mechanical behaviour of nano-beams and carbon nanotubes (we refer again to papers [12]-[18]).

\subsection{Two-phase nonlocal model}

Consider the two-phase nonlocal model (TPNM) which is also called as the local-nonlocal mixed constitutive one. According to Eringen's formulation [30], it is expressed by equation

$$
\sigma_{i j}(\mathbf{x})=\xi_{1} \sigma^{(c)}(\mathbf{x})+\xi_{2} \int_{V} K\left(\left|\mathbf{x}-\mathbf{x}^{\prime}\right|, \varepsilon\right) \sigma_{i j}^{(c)}\left(\mathbf{x}^{\prime}\right) d V\left(\mathbf{x}^{\prime}\right)
$$


where $\xi_{1}$ and $\xi_{2}$ are the volume fractions of the local and non-local phases, respectively. It is clear that $\xi_{1}, \xi_{2}$ are positive constants and $\xi_{1}+\xi_{2}=1$. When $\xi_{1} \rightarrow 0$, Eq. (7) degenerates into (1) corresponding to PNIM, and for $\xi_{1}=1$, one has the constitutive equations

$$
\sigma_{i j}(\mathbf{x}) \equiv \sigma^{(c)}(\mathbf{x})=C_{k l i j} e_{k l}(\mathbf{x})
$$

for the classical (local) elasticity, where $C_{k l i j}$ denotes the components of the fourth-rank tensor of classical isotropic elasticity, and $e_{k l}$ are the components of the strain tensor at $\mathbf{x}$. We will show below that the limiting process at $\varepsilon \rightarrow 0$ also results in the classical constitutive equations, this degeneration being uniform by $\xi_{1}$.

The simplified version of Eq. (7) for 1D problem is used in what follows.

\section{Governing equations and boundary conditions}

We shall consider a uniform rod of length $L$ with Young's modulus $E$, and density $\rho$. Let $x_{1}$ be the coordinate directed along the rod's length, $u$ the longitudinal displacement of the rod's cross section, and $\sigma=\sigma_{11}$ the axial stress corresponding to the linear stress-strain state in the rod. Then, the differential equation governing the small longitudinal vibrations in absence of external load can be written as

$$
\frac{\partial \sigma}{\partial x_{1}}-\rho \frac{\partial^{2} u}{\partial t^{2}}=0
$$

where $t$ is time.

Let $x, \tau$ be the dimensionless counterparts for $x_{1}, t$, respectively, which are introduced as

$$
x_{1}=l_{c} x, \quad t=t_{c} \tau,
$$

where $l_{c}=L$ is the external characteristic size for the beam, and $t_{c}=\sqrt{\frac{\rho l_{c}^{2}}{E}}$ is the characteristic time.

For free vibrations,

$$
u=l_{c} y(x) \exp (\mathrm{i} \omega \tau)
$$

where $\omega$ is a required dimensionless natural frequency.

Assuming one of the above mentioned models and substituting (10), (11) into (9), one obtains the appropriate governing equations in the dimensionless form:

$$
\begin{aligned}
& \text { for PNIM : } \\
& \qquad \begin{array}{c}
\frac{1}{2 \varepsilon} \frac{d}{d x} \int_{0}^{1} R(|x-s|, \varepsilon) y^{\prime}(s) d s+\omega^{2} y=0, \\
\text { for DNM : } \\
\text { for TPNM : } \quad\left(1-\varepsilon^{2} \omega^{2}\right) \frac{d^{2} y}{d x^{2}}+\omega^{2} y=0, \\
\xi_{1} \frac{d^{2} y}{d x^{2}}+\frac{\xi_{2}}{2 \varepsilon} \frac{d}{d x} \int_{0}^{1} R(|x-s|, \varepsilon) y^{\prime}(s) d s+\omega^{2} y=0,
\end{array}
\end{aligned}
$$

where $R(|x-s|, \varepsilon)=K\left(l_{c}|x-s|, \varepsilon\right)$, and the prime $\left\{{ }^{\prime}\right\}$ denotes differentiation by $s$. 
Here we consider three types of boundary conditions, namely, conditions of clamped ends, free ends, and their combination. For clamped ends (CC conditions),

$$
y(0)=y(1)=0,
$$

for free ends (FF conditions),

$$
\sigma(0)=\sigma(1)=0
$$

and for clamped and free ends (CF conditions),

$$
y(0)=\sigma(1)=0 .
$$

It should be noted that the boundary condition for a free edge written in terms of the displacement $y$ depends on the type of a model accepted. Indeed, for the PNIM and TPNM, these conditions are given by

$$
\int_{0}^{1} R(|x-s|, \varepsilon) y^{\prime}(s) d s=0
$$

and

$$
\xi_{1} \frac{d y}{d x}+\frac{\xi_{2}}{2 \varepsilon} \int_{0}^{1} R(|x-s|, \varepsilon) y^{\prime}(s) d s=0,
$$

respectively, at $x=0$ or/and $x=1$. As for the DNM represented by Eq. (13), to state the appropriate condition(s), one needs first to find the stress $\sigma$ from either Eq. (9) or the differential equation

$$
\varepsilon^{2} \frac{d^{2} \sigma}{d x^{2}}-\sigma=-E \frac{d y}{d x}
$$

Obviously, in order to find the natural frequency $\omega$ and the associated eigensolution of (20), an additional boundary condition at the free edge should be derived, depending on the kernel in the original PNIM. Another option is to revert to the original PNIM and satisfy the boundary condition (18).

\section{Brief analysis of nonlocal models. Equivalent differential equation}

Let $e_{0} a \ll l_{c}$, so that a parameter $\varepsilon$ defined by (2) may be considered as a small one. When $\varepsilon \rightarrow 0$, each of the above nonlocal models reverts to the classical local model with the governing equation

$$
\frac{d^{2} y}{d x^{2}}+\omega^{2} y=0
$$

and the boundary conditions $y=0$ and $y^{\prime}=0$ for the clamped and free edges, respectively.

As a preliminary, consider the DNM, which is relatively simple and used frequently by many authors. The brief analysis shows that it is inconsistent, since the total degree of the differential equations (13), (20) is higher then the number of boundary conditions. Indeed, although equation (13) admits the explicit solution

$$
y=c_{1} \sin \lambda x+c_{2} \cos \lambda x,
$$

where

$$
\lambda=\frac{\omega}{\sqrt{1-\varepsilon^{2} \omega^{2}}}
$$


and $c_{1}, c_{2}$ are arbitrary constants, the substitution of (22), (23) into (20) does not allow to find neither stress distribution $\sigma(x)$ for the CC conditions, nor the natural modes and frequencies, if some of the ends are free.

To resolve this contradiction, in many papers the boundary conditions for nano-scale stresses $\sigma_{i j}$ are replaced by conditions for the classical (macroscopic) stresses $\sigma_{i j}^{(c)}$. In particular, when considering bending vibrations of a nanobeam, the boundary conditions for simply supported ends turn out to coincide for both DNM and classical (local) elasticity [12], however, they are different from those in the framework of nonlocal integral models (PNIM and TPNM). If we follow this simplified approach in the framework of DNM, then for the longitudinal vibrations of a nanorod the condition of a free edge $(\sigma=0)$ should be replaced by $y^{\prime}=0$. As will be shown below, this simplification results in ignoring the size effects (the edge effect integrals) in the neighbourhood of the beam edge and may lead to serious errors for a sufficiently short rod.

Now, we shall consider the nonlocal integrals models (PNIM and TPNM) with the exponential kernel

$$
R(|x-s|, \varepsilon)=\frac{1}{2 \varepsilon} e^{-\frac{|x-s|}{\varepsilon}}
$$

which is the most commonly used for one-dimensional problems, see e.g. [20, 21], [33] -[37]. Since PNIM is the limiting case of TPNM as $\xi_{1} \rightarrow 0$, the subsequent transformations will be performed for equation (14).

Note that

$$
\frac{d}{d x} \int_{0}^{1} e^{-\frac{|x-s|}{\varepsilon}} z(s) d s=\frac{1}{\varepsilon}\left[e^{\frac{x}{\epsilon}} \int_{0}^{1} e^{-\frac{s}{\varepsilon}} z(s) d s-e^{-\frac{x}{\epsilon}} \int_{0}^{1} e^{\frac{s}{\varepsilon}} z(s) d s\right]
$$

and

$$
\frac{d^{2}}{d x^{2}} \int_{0}^{1} e^{-\frac{|x-s|}{\varepsilon}} z(s) d s=\frac{1}{\varepsilon^{2}} \int_{0}^{1} e^{-\frac{|x-s|}{\varepsilon}} z(s) d s-\frac{2}{\varepsilon} z(x) .
$$

Assume the solution $y(x)$ is sought on the set $y(x) \in C^{4}[0,1]$. Differentiating equation (14) twice and taking into account equations (24)-(26), one obtains the integro-differential equation

$$
\varepsilon^{2} \xi_{1} \frac{d^{4} y}{d x^{4}}+\frac{\xi_{2}}{2 \varepsilon} \frac{d}{d x} \int_{0}^{1} e^{-\frac{|x-s|}{\varepsilon}} y^{\prime}(s) d s-\xi_{2} \frac{d^{2} y}{d x^{2}}+\varepsilon^{2} \omega^{2} \frac{d^{2} y}{d x^{2}}=0 .
$$

Using (14) to exclude the second term in (27), we arrive at the following differential equation of the fourth order

$$
\varepsilon^{2} \xi_{1} \frac{d^{4} y}{d x^{4}}-\left(1-\varepsilon^{2} \omega^{2}\right) \frac{d^{2} y}{d x^{2}}-\omega^{2} y=0,
$$

which will serve as the governing one in our analysis. It should be noted that the same fourth-order equation (28) has been obtained by Challamel [20,34] for a special exponential kernel different from (24).

Using equation (25), one can rewrite the boundary conditions (16) for free edges. They read as

$$
\varepsilon \xi_{1} y^{\prime}(0)+\frac{1-\xi_{1}}{2} \int_{0}^{1} e^{-\frac{s}{\varepsilon}} y^{\prime}(s) d s=0
$$

and

$$
\varepsilon \xi_{1} y^{\prime}(1)+\frac{\left(1-\xi_{1}\right) e^{-\frac{1}{\varepsilon}}}{2} \int_{0}^{1} e^{\frac{s}{\varepsilon}} y^{\prime}(s) d s=0
$$


for the ends $x=0$ and $x=1$, respectively. If both ends are clamped, then the boundary conditions are given by (15).

The number of listed boundary conditions is not sufficient to solve the above boundary-value problems. The additional conditions may be readily obtained from the initial integro-differential equation (14):

$$
\begin{gathered}
\varepsilon^{2} \xi_{1} y^{\prime \prime}(0)+\frac{1-\xi_{1}}{2} \int_{0}^{1} e^{-\frac{s}{\varepsilon}} y^{\prime}(s) d s+\varepsilon^{2} \omega^{2} y(0)=0, \\
\varepsilon^{2} \xi_{1} y^{\prime \prime}(1)-\frac{\left(1-\xi_{1}\right) e^{-\frac{1}{\varepsilon}}}{2} \int_{0}^{1} e^{\frac{s}{\varepsilon}} y^{\prime}(s) d s+\varepsilon^{2} \omega^{2} y(1)=0 .
\end{gathered}
$$

Note that conditions (31), (32) are not natural boundary conditions. They reflect the internal nonlocal effects near the edges. Another variant of the additional boundary conditions associated with the special kernel in the constitutive equation (7) have been derived in [34], see Eq.(41) in the cited paper.

It is seen that for $\xi_{1}=0$ the governing equation (28) coincides with equation (13) corresponding to DNM. As for conditions (29)-(30), they revert to the boundary conditions for PNIM, which do not coincide with the boundary conditions for DNM, and also become inconsistent with the additional conditions (31), (32) at $\xi_{1}=0$. Thus, within the framework of PNIM $\left(\xi_{1}=0\right)$, the governing equation (27) with appropriate boundary conditions (15) or (29), (30) does not have a solution in $C^{4}[0,1]$. It should be noted that the revealed defect of the dynamical PNIM model has been recently proved by Romano et al. [29] addressing static problems for beam bending.

\section{Exact and asymptotic solutions of the boundary-value problem}

\subsection{Exact solution in the TPNM framework}

Consider the differential equation (28). It is clear that for $y(x) \in C^{4}[0,1]$, it is equivalent to the integrodifferential equation (14). For any $\xi_{1}, \varepsilon>0$, this equation has a straightforward exact solution

$$
y=c_{1} \sin \alpha x+c_{2} \cos \alpha x+c_{3} e^{-\beta x}+c_{4} e^{\beta(x-1)},
$$

where

$$
\begin{aligned}
& \alpha=\sqrt{\frac{\sqrt{\left(1-\varepsilon^{2} \omega^{2}\right)^{2}+4 \varepsilon^{2} \xi_{1} \omega^{2}}-\left(1-\varepsilon^{2} \omega^{2}\right)}{2 \varepsilon^{2} \xi_{1}},} \\
& \beta=\sqrt{\frac{\sqrt{\left(1-\varepsilon^{2} \omega^{2}\right)^{2}+4 \varepsilon^{2} \xi_{1} \omega^{2}}+\left(1-\varepsilon^{2} \omega^{2}\right)}{2 \varepsilon^{2} \xi_{1}}},
\end{aligned}
$$

and $c_{i}$ are constants to be determined from the boundary conditions. We note that the third and fourth terms in (33) describe the edge effects, which are not taken into account within the DNM.

On substitution of (33), (34) into one of the boundary conditions considered, we result in a relation

$$
F\left(\omega, \xi_{1}, \varepsilon\right)=0
$$

with respect to the eigen-frequency $\omega$. As $\xi_{1} \rightarrow 0$, we have $\alpha \rightarrow \lambda$ and $\beta \rightarrow \infty$, where $\lambda$ is defined by (23), and solution (33) transforms to a solution corresponding to PNIM. However, this limiting passage may lead to considerable computational difficulties when solving the transcendental frequency equation (35) containing small parameters. 


\subsection{Asymptotic solutions (TPNM)}

Since $\varepsilon$ is a small parameter, the differential equation (27) is a singularly perturbed one, corresponding to (21) at $\varepsilon \rightarrow 0$. In this subsection we construct asymptotic solutions of equation (27) for all studied types of boundary conditions at $\varepsilon \rightarrow 0$. These solutions will provide simple estimates for natural frequencies, allow the limiting process at $\xi_{1} \rightarrow 0$ and $\varepsilon \rightarrow 0$ and also provide a foundation for comparison of different nonlocal models as well.

We seek the solution in the form of superposition of the two functions, namely $y_{m}(x)$ and $y_{e}(x)$, corresponding to the main stress state and the edge effect integrals, respectively

$$
y=y_{m}(x)+\varepsilon^{\gamma} y_{e}(x),
$$

where $\gamma$ is the index of intensity of the edge effect integrals accounting for the influence of non-locality near the edges. As will be shown below, the value of $\gamma$ depends on the type of boundary conditions assumed. The procedure for the index of intensity of the classical edge effect integrals in thin shells is described in details in [45].

The functions $y_{m}(x), y_{e}(x)$ and required eigenvalue $\omega$ are expanded as asymptotic series

$$
\begin{gathered}
y_{m}=y_{m 0}+\varepsilon y_{m 1}+\varepsilon^{2} y_{m 2}+\ldots, \\
y_{e}=y_{e 0}+\varepsilon y_{e 1}+\varepsilon^{2} y_{e 2}+\ldots, \\
\omega=\omega_{0}+\varepsilon \omega_{1}+\varepsilon^{2} \omega_{2}+\ldots
\end{gathered}
$$

It is assumed that

$$
\frac{d y_{m k}}{d x} \sim y_{m k}(x), \quad \frac{d y_{e k}}{d x} \sim \varepsilon^{-\tau} y_{e k}(x) \quad \text { at } \quad \varepsilon \rightarrow 0 \quad \text { for any } \quad x \in[0,1],
$$

where $\tau>0$ is an unknown index of variation of edge effect integrals.

To derive an equation accounting for the edge effects, one needs to assume $x=\varepsilon^{\tau} \zeta$ and $x=1+\varepsilon^{\tau} \zeta$ near the left and right ends of the rod, respectively. In both cases, transforming to a new variable $\zeta$ gives $\tau=1$ and results in the following differential equation

$$
\xi_{1} \frac{d^{4} y_{e}}{d \zeta^{4}}-\left(1-\varepsilon^{2} \omega^{2}\right) \frac{d^{2} y_{e}}{d \zeta^{2}}-\varepsilon^{2} \omega^{2} y_{e}=0
$$

which is the same for both ends.

Inserting series (38) and (39) into (41) generates a perturbation procedure with respect to $y_{e k}(\zeta)$. The resulting two-term solution in terms of the original variable $x$ is given by

$$
\begin{aligned}
& y_{e}=a_{01} e^{-\frac{x}{\varepsilon \sqrt{\xi_{1}}}}+a_{02} e^{\frac{x-1}{\varepsilon \sqrt{\xi_{1}}}}+\varepsilon\left[a_{11} e^{-\frac{x}{\varepsilon \sqrt{\xi_{1}}}}+a_{12} e^{\frac{x-1}{\varepsilon \sqrt{\xi_{1}}}}+\right. \\
& \left.\frac{\omega_{0}^{2}\left(1-\xi_{1}\right)}{2 \sqrt{\xi_{1}^{3}}}\left(a_{01} x e^{-\frac{x}{\varepsilon \sqrt{\xi_{1}}}}-a_{02}(x-1) e^{\frac{x-1}{\varepsilon \sqrt{\xi_{1}}}}\right)\right]+O\left(\varepsilon^{2}\right) .
\end{aligned}
$$

The function $y_{m}(x)$ corresponding to the main stress state may be found from substitution of the series (37), (39) into the governing equation (28). Equating coefficients by the same powers of $\varepsilon$, we 
deduce

$$
\begin{gathered}
\mathbf{L}_{0} y_{m 0}=\frac{d^{2} y_{m 0}}{d x^{2}}+\omega^{2} y_{m 0}=0, \\
\mathbf{L}_{0} y_{m 1}=-2 \omega_{0} \omega_{1} y_{m 0}, \\
\mathbf{L}_{0} y_{m 2}=-2 \omega_{0} \omega_{1} y_{m 1}+\xi_{1} \frac{d^{4} y_{m 0}}{d x^{4}}+\omega_{0}^{2} \frac{d^{2} y_{m 0}}{d x^{2}}-\left(2 \omega_{0} \omega_{2}+\omega_{1}^{2}\right) y_{m 0}, \ldots .
\end{gathered}
$$

Note that (43) is a homogeneous equation of second order, whereas there are four boundary conditions. Hence, one needs to split the boundary conditions and assign the two main conditions for $y_{m 0}$, with the additional ones used to determine $y_{m k}$ for $k \geq 1$ and the constants $a_{k 1}, a_{k 2}$ appearing in (42). To this purpose, we substitute (36), (37) and (42) into the boundary conditions and, taking into account the asymptotic estimates (40), impose the following conditions:

- the main boundary conditions for $y_{m 0}(x)$ should be homogeneous;

- for any $k=0,2, \ldots$, there are equations coupling $a_{k 1}, a_{k 2}$ with $y_{m k}^{\prime}(0), y_{m k}^{\prime}(1)$;

- for any $k=1,2, \ldots$, there is an inhomogeneous equation for $y_{m k}(x)$ at each of the ends.

In what follows, a detailed consideration is carried out only for one type of boundary conditions.

Let both ends be clamped (C-C conditions) with the associated boundary conditions (15), (31) and (32). Taking into account the above requirements, one obtains the index of intensity for the edge effect integrals, $\gamma=1$, with the main boundary conditions taking the form

$$
y_{m 0}(0)=y_{m 0}(1)=0 \text {. }
$$

In the first-order approximation, conditions (15) give

$$
y_{m 1}(0)=-y_{e 0}(0)=-a_{01}, \quad y_{m 1}(1)=-y_{e 0}(1)=-a_{02},
$$

and the additional boundary conditions (31), (32) result in

$$
\begin{aligned}
& \left(1-\sqrt{\xi_{1}}\right) y_{m 0}^{\prime}(0)+a_{01}=0, \\
& \left(1-\sqrt{\xi_{1}}\right) y_{m 0}^{\prime}(1)-a_{02}=0 .
\end{aligned}
$$

The second-order approximation yields

$$
y_{m 2}(0)=-a_{11}, \quad y_{m 2}(1)=-a_{12},
$$

and

$$
\begin{aligned}
& \left(1-\sqrt{\xi_{1}}\right)\left[y_{m 1}^{\prime}(0)+y_{m 0}^{\prime \prime}(0)\right]+a_{11}=0, \\
& \left(1-\sqrt{\xi_{1}}\right)\left[y_{m 1}^{\prime}(1)+y_{m 0}^{\prime \prime}(1)\right]-a_{12}=0 .
\end{aligned}
$$

When deriving relations (48) and (50), we have taken into account relations (40) and the holding asymptotic estimates for integrals:

$$
\begin{aligned}
& \int_{0}^{1} e^{-\frac{s}{\varepsilon}} y_{m}^{\prime}(s) d s=\varepsilon\left[y_{m}^{\prime}(0)+\varepsilon y_{m}^{\prime \prime}(0)+\varepsilon^{2} y_{m}^{\prime \prime \prime}(0)+O\left(\varepsilon^{3}\right)\right], \\
& \int_{0}^{1} e^{\frac{s}{\varepsilon}} y_{m}^{\prime}(s) d s=\varepsilon e^{\frac{1}{\epsilon}}\left[y_{m}^{\prime}(1)-\varepsilon y_{m}^{\prime \prime}(1)+\varepsilon^{2} y_{m}^{\prime \prime \prime}(1)+O\left(\varepsilon^{3}\right)\right] .
\end{aligned}
$$


Furthermore, when calculating integrals in (31), (32), the terms of $\varepsilon^{k} e^{-\frac{1}{\varepsilon}}$ for any $k$ were neglected.

Consider now boundary-value problems arising in the first three asymptotic orders.

In the leading order approximation, the classical homogeneous boundary-value problem (43), (46), corresponding to local elasticity, results in

$$
y_{m 0}(x)=C \sin \omega_{0} x, \quad \omega_{0}=\pi n, \quad n=1,2, \ldots,
$$

where $C$ is an arbitrary constant.

In the first-order approximation, one obtains

$$
a_{01}=-\pi n\left(1-\sqrt{\xi_{1}}\right) C, \quad a_{01}=(-1)^{n} \pi n\left(1-\sqrt{\xi_{1}}\right) C
$$

and the boundary conditions

$$
y_{m 1}(0)=\pi n\left(1-\sqrt{\xi_{1}}\right) C, \quad y_{m 1}(0)=(-1)^{n+1} \pi n\left(1-\sqrt{\xi_{1}}\right) C
$$

for the non-homogeneous equation (44). The existence of solution of the eigenvalue problem (44), (54) implies a correction for the eigenfrequency

$$
\omega_{1}=-2 \pi n\left(1-\sqrt{\xi_{1}}\right) .
$$

Then,

$$
y_{m 1}(x)=C \pi n\left(1-\sqrt{\xi_{1}}\right)(1-2 x) \cos \pi n x,
$$

and equations (50) give

$$
a_{11}=2 \pi n\left(1-\sqrt{\xi_{1}}\right)^{2} C, \quad a_{12}=(-1)^{n+1} 2 \pi n\left(1-\sqrt{\xi_{1}}\right)^{2} C .
$$

Now, we can consider the next order boundary-value problem (45), (49). The compatibility condition of this problem leads to the next approximation for the eigenfrequency

$$
\omega_{2}=4 \pi n\left(1-\sqrt{\xi_{1}}\right)^{2}-\frac{1}{2}(\pi n)^{3}\left(1-\xi_{1}\right) .
$$

The following approximations may be constructed in a similar manner. Thus, we can write down the following approximate formulas for the dimensional natural frequencies

$$
\omega=\pi n\left\{1-2 \varepsilon\left(1-\sqrt{\xi_{1}}\right)+\varepsilon^{2}\left[4\left(1-\sqrt{\xi_{1}}\right)^{2}-\frac{1}{2}(\pi n)^{2}\left(1-\xi_{1}\right)\right]+O\left(\varepsilon^{3}\right)\right\},
$$

and modes

$$
\begin{aligned}
y(x)=\sin \pi n x+\varepsilon \pi n & \left(1-\sqrt{\xi_{1}}\right)[(1-2 x) \cos \pi n x- \\
& \left.e^{-\frac{x}{\varepsilon \sqrt{\xi_{1}}}}+(-1)^{n} e^{\frac{x-1}{\varepsilon \sqrt{\xi_{1}}}}\right]+O\left(\varepsilon^{2}\right), \quad n=1,2, \ldots,
\end{aligned}
$$


with normalized amplitudes.

Similar asymptotic considerations for the remaining two types of boundary conditions lead to

$$
\begin{aligned}
& \omega=\pi\left(\frac{1}{2}+n\right)\left\{1-\varepsilon\left(1-\sqrt{\xi_{1}}\right)-\right. \\
& \left.\frac{1}{2} \varepsilon^{2}\left(1-\sqrt{\xi_{1}}\right)\left[\left(\frac{1}{2}+n\right)^{2}\left(1+\sqrt{\xi_{1}}\right)-2\left(1-\sqrt{\xi_{1}}\right)\right]+O\left(\varepsilon^{3}\right)\right\}, \\
& y(x)=\sin \pi\left(\frac{1}{2}+n\right) x+\varepsilon \pi\left(\frac{1}{2}+n\right)\left(1-\sqrt{\xi_{1}}\right) \times \\
& {\left[(1-x) \cos \pi\left(\frac{1}{2}+n\right) x-e^{-\frac{x}{\varepsilon \sqrt{\xi_{1}}}}\right]+O\left(\varepsilon^{2}\right), \quad n=0,1,2, \ldots}
\end{aligned}
$$

and

$$
\begin{aligned}
& \omega=\pi n\left[1-\frac{1}{2} \varepsilon^{2}(\pi n)^{2}\left(1-\xi_{1}\right)+O\left(\varepsilon^{3}\right)\right], \quad n=0,1,2, \ldots \\
& y(x)=\cos \pi n x-\varepsilon^{2}(\pi n)^{2}\left(1-\sqrt{\xi_{1}}\right)\left[e^{-\frac{x}{\varepsilon \sqrt{\xi_{1}}}}+(-1)^{n} e^{\frac{x-1}{\varepsilon \sqrt{\xi_{1}}}}\right]+O\left(\varepsilon^{3}\right)
\end{aligned}
$$

for the CF and FF conditions, respectively.

It is seen, that for both the $\mathrm{CC}$ and $\mathrm{CF}$ rods, $\gamma=1$, while for the FF conditions, one has $\gamma=2$. In other words, in the first two cases (for CC and CF conditions) the corrections induced by the nonlocal effect are of order $O(\varepsilon)$, and for rods with free ends it is smaller, being of order $O\left(\varepsilon^{2}\right)$.

At $\xi_{1} \rightarrow 1$ or $\varepsilon \rightarrow 0$, Eqs. (59)-(62) degenerate into the well-known simple formulas for the classical (local) model. It is important to note that this limiting process is uniform by $\varepsilon$ and $\xi_{1}$ (if $\xi_{1} \rightarrow 1$ and $\varepsilon \rightarrow 0$, respectively). This property provides the asymptotic justification of TPNM when comparing it to the classical "local" elasticity theory.

It is of interest to compare the eigenfrequencies (59) and the associated modes (60) with the analogous solution obtained by Challamel [34] for the CC nanorod. Assuming the special kernel

$$
R(x, s)= \begin{cases}\frac{\cosh \left(\frac{1-s}{\varepsilon}\right)}{\varepsilon \sinh \left(\frac{1}{\varepsilon}\right)} \cosh \left(\frac{x}{\varepsilon}\right), & \text { if } \quad x \leq s, \\ \frac{\cosh \left(\frac{1-x}{\varepsilon}\right)}{\varepsilon \sinh \left(\frac{1}{\varepsilon}\right)} \cosh \left(\frac{s}{\varepsilon}\right), & \text { if } \quad x \geq s\end{cases}
$$

it was found that

$$
\begin{aligned}
& \omega=\pi n \sqrt{\frac{1+\varepsilon^{2} \xi_{1}(\pi n)^{2}}{1+\varepsilon^{2}(\pi n)^{2}}}=\pi n\left[1-\frac{1}{2} \varepsilon^{2}\left(1-\xi_{1}\right)(\pi n)^{2}+O\left(\varepsilon^{4}\right)\right], \\
& y(x)=\sin (\pi n x) .
\end{aligned}
$$

It may be observed that the result strongly depends on the assumed kernel in the constitutive equation. In our case, for the exponential kernel (24), the correction to the eigenfrequencies found in the framework of the classical elasticity theory is the value of order $O(\varepsilon)$, while for the special kernel (63), it is of order $O\left(\varepsilon^{2}\right)$. 


\subsection{Asymptotic solutions in the PNIM framework}

Although the asymptotic solutions (59)-(62) are valid for positive values of $\xi_{1}$, it is of interest to consider the limiting case (corresponding to PNIM) at $\xi_{1} \rightarrow 0$. Eqs. (59)-(62) become

$$
\begin{aligned}
& \omega=\pi n\left\{1-2 \varepsilon+\varepsilon^{2}\left[4-\frac{1}{2}(\pi n)^{2}\right]+O\left(\varepsilon^{3}\right)\right\}, \quad n=1,2, \ldots, \\
& y=\sin \pi n x+\varepsilon \pi n(1-2 x) \cos \pi n x+O\left(\varepsilon^{2}\right)
\end{aligned}
$$

for $\mathrm{CC}$ conditions,

$$
\begin{aligned}
& \omega=\pi\left(\frac{1}{2}+n\right)\left\{1-\varepsilon-\frac{1}{2} \varepsilon^{2}\left[\left(\frac{1}{2}+n\right)^{2}-2\right]+O\left(\varepsilon^{3}\right)\right\}, \quad n=0,1,2, \ldots, \\
& y=\sin \pi\left(\frac{1}{2}+n\right) x+\varepsilon \pi\left(\frac{1}{2}+n\right)(1-x) \cos \pi\left(\frac{1}{2}+n\right) x+O\left(\varepsilon^{2}\right)
\end{aligned}
$$

for $\mathrm{CF}$ conditions, and

$$
\begin{aligned}
& \omega=\pi n\left[1-\frac{1}{2} \varepsilon^{2}(\pi n)^{2}+O\left(\varepsilon^{3}\right)\right], \quad n=0,1,2, \ldots, \\
& y=\cos \pi n x+O\left(\varepsilon^{3}\right)
\end{aligned}
$$

and for FF conditions.

\subsection{Solutions in the DNM framework}

Consider Eq. (13) related to DNM. Its asymptotic solutions are

$$
\begin{aligned}
& \omega=\frac{\pi n}{\sqrt{1+\varepsilon^{2}(\pi n)^{2}}}=\pi n\left[1-\frac{1}{2} \varepsilon^{2}(\pi n)^{2}+O\left(\varepsilon^{4}\right)\right], \quad n=1,2, \ldots, \\
& y=\sin \frac{\omega x}{\sqrt{1-\varepsilon^{2} \omega^{2}}}
\end{aligned}
$$

for rods with CC conditions,

$$
\begin{aligned}
& \omega=\frac{\pi\left(\frac{1}{2}+n\right)}{\sqrt{1+\varepsilon^{2} \pi^{2}\left(\frac{1}{2}+n\right)^{2}}}=\pi\left(\frac{1}{2}+n\right)\left[1-\frac{1}{2} \varepsilon^{2} \pi^{2}\left(\frac{1}{2}+n\right)^{2}+O\left(\varepsilon^{4}\right)\right], \quad n=0,1,2, \ldots, \\
& y=\sin \frac{\omega x}{\sqrt{1-\varepsilon^{2} \omega^{2}}}
\end{aligned}
$$

for CF conditions, and

$$
\begin{aligned}
& \omega=\frac{\pi n}{\sqrt{1+\varepsilon^{2}(\pi n)^{2}}}=\pi n\left[1-\frac{1}{2} \varepsilon^{2}(\pi n)^{2}+O\left(\varepsilon^{4}\right)\right], \quad n=0,1,2, \ldots, \\
& y=\cos \frac{\omega x}{\sqrt{1-\varepsilon^{2} \omega^{2}}}
\end{aligned}
$$

for the FF case.

Note that in the process of derivation of results (69) and (70), we have assumed $y^{\prime}=0$ at the free ends as it has been, particularly, made in paper [16]. We note also that formulas (69) were obtained before by Aydogdu [16] on the basis of the DNM.

In the next Section, we compare and discuss the above solutions found within the framework of different nonlocal models. 
(a)

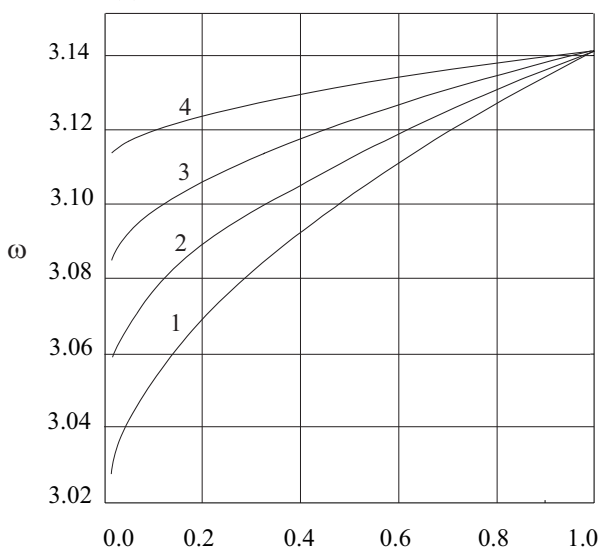

(b)

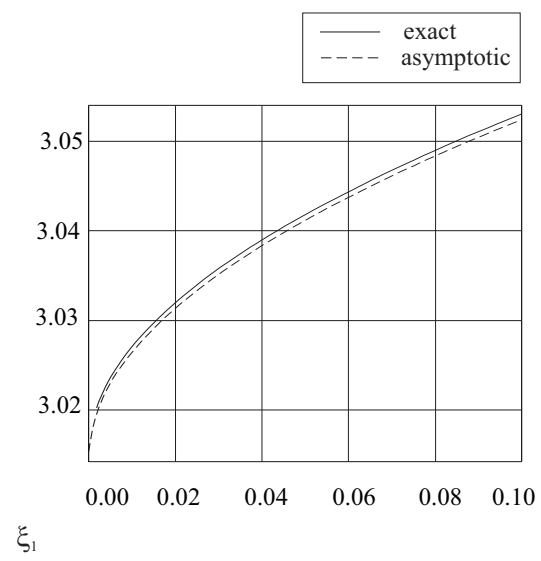

Figure 1. The lowest dimensionless frequency $\omega$ (at $n=1$ ) of the rod with CC ends vs. parameter $\xi_{1}$ :

(a) exact results for different values of $\varepsilon$ : $1-\varepsilon=0.02,2-\varepsilon=0.015,3-\varepsilon=0.01,4-\varepsilon=0.005$;

(b) comparison of exact and asymptotic results for $\varepsilon=0.02$

\section{Analysis and discussions}

The common and expected conclusion following from the aforementioned solutions is that incorporation of the nonlocal parameter $\varepsilon$ results in a decrease in eigenfrequencies $\omega$ for any nonlocal model. However, the nature of this effect is different for the so-called "equivalent" differential model and integral ones. The approach based on DNM allows taking into account the general nonlocal effect only, involving the correction of order $O\left(\varepsilon^{2}\right)$ to the classical eigenvalues, while the integral models (PNIM and TPNM) allow incorporation of the nonlocal edge effects and give the contribution of order $O(\varepsilon)$. Thus, the DNM, which is used rather frequently, gives overestimated results. Also, simple solutions (69) and (70) for a rod with one or two free ends do not satisfy the real boundary conditions in terms of stresses (see Eqs. (29), (30) at $\left.\xi_{1}=0\right)$. Obviously, an error of results obtained on the basis of DNM may be enourmous if a nanorod is sufficiently short. This conclusion on inconsistency of DNM is also confirmed by results of [22], studying the dynamic response of a non-locally elastic half-space with a traction-free surface.

As for PNIM, solutions (65) and (66) satisfy the boundary conditions of clamped ends with accuracy of $O(\varepsilon)$, and modes (66) and (67) obtained for the rods with one and two free edges, respectively, do not satisfy the additional conditions (31), (32). Thus, the purely nonlocal integral model (PNIM) also turns out to be ill-posed.

To analyse further the results based on TPNM, numerical illustrations of the dimensionless natural frequencies $\omega$ for rods with different boundary conditions are presented below. Figs. 1(a), 2(a), and 3(a) show exact values of the lowest eigenvalue $\omega$, found from equation (35) for the $\mathrm{CC}$, $\mathrm{CF}$ and FF conditions, versus the volume fraction $\xi_{1} \in(0,1]$ and different scale parameters $\varepsilon=0.02,0.015,0.005$. The assumed values of $\varepsilon$ were calculated at $e_{0}=0.39, a=0.142 \mathrm{~nm}$ associated with a carbon nanotube and different lengths $l_{c}$ varying from 2 to $10 \mathrm{~nm}$. Figs. 1(b), 2(b), and 3(b) display comparison of asymptotic results obtained from (59), (61), (62) against exact solutions of equation (35) for $\varepsilon=0.02$. 
(a)

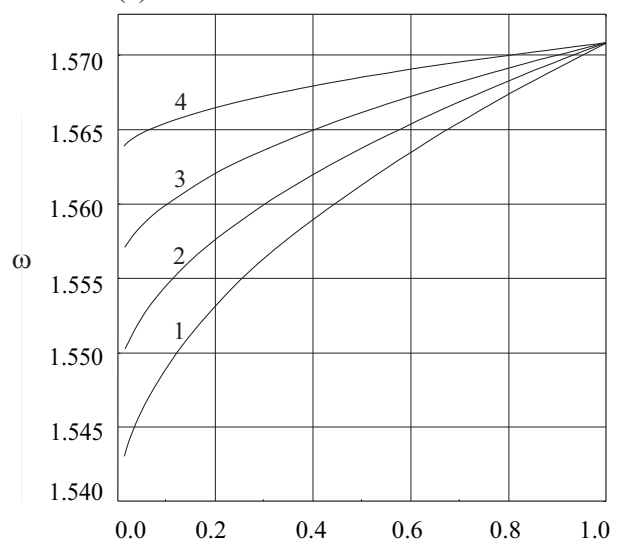

(b)

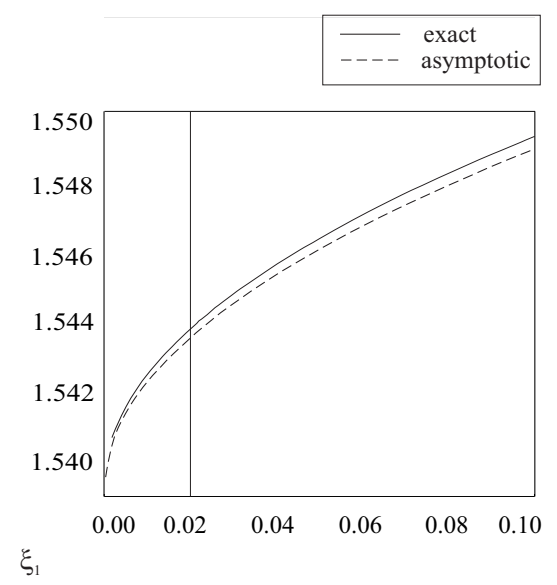

Figure 2. The lowest dimensionless frequency $\omega$ (at $n=0$ ) of the rod with CF ends vs. parameter $\xi_{1}$ : (a) exact results for different values of $\varepsilon$ : $1-\varepsilon=0.02,2-\varepsilon=0.015,3-\varepsilon=0.01,4-\varepsilon=0.005$;

(b) comparison of exact and asymptotic results for $\varepsilon=0.02$

(a)

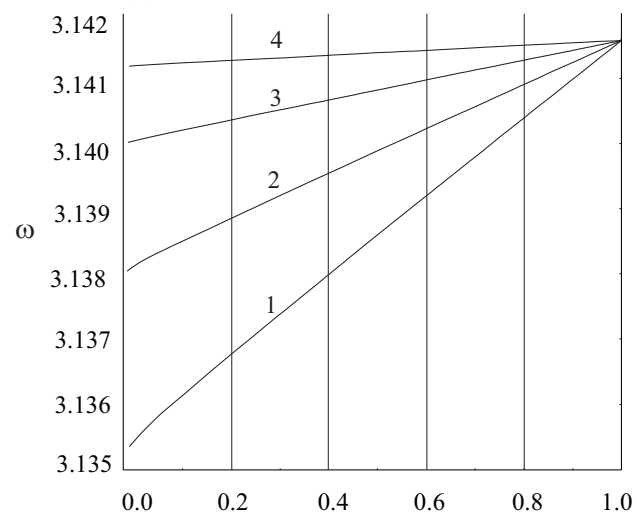

(b)

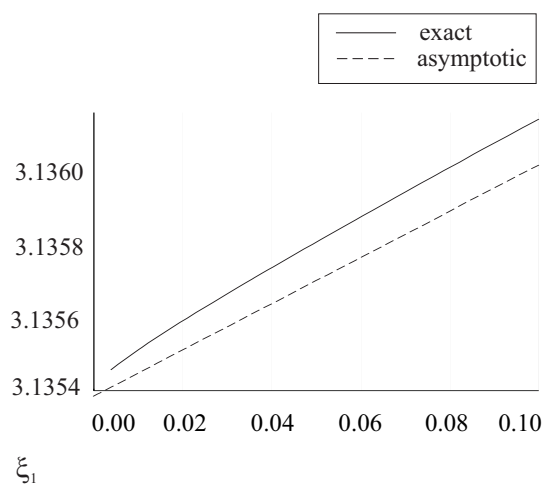

Figure 3. The lowest dimensionless frequency $\omega$ (at $n=1$ ) of the rod with FF ends vs. parameter $\xi_{1}$ :

(a) exact results for different values of $\varepsilon$ : $1-\varepsilon=0.02,2-\varepsilon=0.015,3-\varepsilon=0.01,4-\varepsilon=0.005$;

(b) comparison of exact and asymptotic results for $\varepsilon=0.02$

Although the exact and asymptotic solutions are plotted only in the small interval $\xi_{1} \in(0,0.1]$, the comparative analysis of calculations performed for any $\xi_{1} \in(0,1]$ shows that the relative error of the asymptotic formulas is very small. For example, for $\varepsilon=0.02$, it is equal to $0.022,0.025,0.004 \%$ for the $\mathrm{CC}, \mathrm{CF}$ and $\mathrm{FF}$ conditions, respectively. 
It is observed that the eigenvalue $\omega$ decreases together with the volume fraction $\xi_{1}$. Thus, the PNIM gives the lowest values of eigenfrequencies in comparison with TPNM for any type of boundary conditions.

\section{Conclusions}

The problem of free longitudinal vibrations of an elastic nanorod, has been examined within the framework of several nonlocal Eringen's constitutive models (TPNM, PNIM and DNM). First, assuming the exponential attenuation kernel in the Eringen's nonlocal integral models, we have reduced the integrodifferential equation governing vibrations of a nanorod in the TPNM framework to the differential equation of the fourth order. Considering three different types of boundary conditions, namely, clampedclamped, clamped-free and free-free ends, we have supplemented those with two additional conditions accounting for nonlocality near the ends. The analytical exact solutions, as well as the asymptotic ones, have been derived for all three types of the boundary conditions.

Comparison of the results has revealed that natural modes for rods with $\mathrm{CF}$ and FF boundary conditions, found within the DNM framework, do not satisfy the realistic free end boundary conditions and do not take into account the edge effect near a clamped end. Moreover, the solutions relying on PNIM do not satisfy the additional boundary conditions, following from the governing integro-differential equation. Also, the natural modes found in the PNIM framework satisfy the boundary condition of a clamped edge only approximately, with the error of order $O(\varepsilon)$. Thus, both approaches based on the purely nonlocal Eringen's stress theory (PNIM) and its "equivalent" differential analogue (DNM) are inconsistent and give erroneous results in the analysis of free vibrations of nanorods.

The numerical calculations performed for different values of a scale parameter $\varepsilon$, predictably revealed that for any type of boundary conditions the natural frequencies are monotonically increasing functions of the volume fraction $\xi_{1}$ corresponding to the classical (local) component in the two-phase nonlocal Eringen's stress theory model. The important result of our study is that when $\varepsilon \rightarrow 0$, then all the natural frequencies and modes degenerate into results corresponding to the classical elasticity theory for any $\xi_{1} \in(0,1]$, and and vice versa, this limiting process at $\xi_{1} \rightarrow 1$ is valid for any $\varepsilon$. That means that TPNM degenerates into the classical model uniformly with respect to both parameters $\varepsilon$ and $\xi_{1}$ and allows a conclusion that the two-phase nonlocal Eringen's stress theory is asymptotically consistent.

Finally, we remark that the analytical and asymptotic solutions constructed in this work for the simplest case of a nanorod, may be considered as a benchmark for subsequent investigations of vibrations of nanosized beams, plates and shells.

\section{Funding}

GM and DP acknowledge the Erasmus+ financial support, allowing visits of GM to Keele University. 


\section{References}

[1] Kröner, E. Elasticity theory of materials with long range cohesive forces. Int J Solids Struct 1967; 3(5): 731-742.

[2] Kunin, IA. The theory of elastic media with microstructure and the theory of dislocation. In: Kröner, E (ed) Mechanics of Generalized Continua. Proceedings of IUTAM Symposium. Berlin, Heidelberg: Springer-Verlag, 1968.

[3] Krumhansl, JA. Some considerations on the relations between solid state physics and generalized continuum mechanics. In: Kröner, E (ed) Mechanics of Generalized Continua. Proceedings of IUTAM Symposium. Berlin, Heidelberg: Springer-Verlag, 1968.

[4] Edelen, DGB. Protoelastic bodies with large deformation. Arch Ration Mech Anal 1969; 34(4): 283-300.

[5] Edelen, DGB, and Laws, N. On the thermodynamics of systems with nonlocality. Arch Ration Mech Anal 1971; 43(1): 24-35.

[6] Eringen, AC. Nonlocal polar elastic continua. Int J Eng Sci 1972; 10(1): 1-16.

[7] Eringen, AC, and Edelen, DGB. On nonlocal elasticity. Int J Eng Sci 1972; 10(3): 233-248.

[8] Eringen, AC. On differential equations of nonlocal elasticity and solutions of screw dislocation and surface waves. J Appl Phys 1983; 54(9): 4703-4710.

[9] Eringen, AC. Nonlocal continuum field theories. New York: Springer, 2002.

[10] Peddieson, J, Buchanan, R, and McNitt, RP. Application of nonlocal continuum models to nanotechnology. Int J Eng Sci 2003; 41(3): 305-312.

[11] Sudak, LJ. Column buckling of multiwalled carbon nanotubes using nonlocal con-tinuum mechanics. J Appl Phys 2003; 94(11): 7281-7287.

[12] Reddy, JN. Nonlocal theories for bending, buckling and vibration of beams. Int J Eng Sci 2007; 45(2): 288-307.

[13] Lu, P, Zhang, PQ, Lee, HP, et al. Non-local elastic plate theories. Proc Royal Soc A 2009; 463 : 3225-3240.

[14] Usuki, T, and Yogo, K. Beam equations for multi-walled carbon nanotubes derived from Flügge shell theory. Proc Royal Soc A 2009; 465: 1199-1226.

[15] Wang, CY, Ru, CQ, and Mioduchowski, A. Free vibrations of multiwalled carbon nanotube. J Appl Phys 2005; 97(11): 114323.

[16] Aydogdu, M. Axial vibration of the nanorods with the nonlocal continuum rod model. Phys E 2009; 41: 861-864. 
[17] Zhang, YQ, Liu, GR, and Xie, XY. Free transverse vibrations of double-walled carbon nanotubes using a theory of nonlocal elasticity. Phys Rev B 2005; 71(19): 195404.

[18] Xu, M. Free transverse vibrations of nano-to-micron scale beams. Proc Royal Soc A 2006; 462: 2977-2995.

[19] Wang, Q, and Liew, KM. Application of nonlocal continuum mechanics to static analysis of microand nano-structures. Phys Lett A 2007; 363(3): 236-242.

[20] Challamel, N, and Wang, CM. The small length scale effect for a non-local cantilever beam: a paradox solved. Nanotechnology 2008; 19(34): 345703.

[21] Benvenuti, E, and Simone, A. One-dimensional nonlocal and gradient elasticity: closed-form solution and size effect. Mech Res Commun 2013; 48: 46-51.

[22] Chebakov, R, Kaplunov, J, and Rogerson, GA. Refined boundary conditions on the free surface of an elastic half-space taking into account non-local effects. Proc Royal Soc A 2016; 472(2186): 20150800.

[23] Chebakov, R, Kaplunov, J, and Rogerson, GA. A non-local asymptotic theory for thin elastic plates. Proc Royal Soc A 2017; 473(2203): 20170249.

[24] Fernández-Sáez, J, Zaera, R, Loya, JA, et al. Bending of Euler-Bernoulli beams using Eringen's integral formulation: paradox resolved. Int J Eng Sci 2016; 99: 107-116.

[25] Polyanin, A, and Manzhirov, A. Handbook of integral equations. New York: CRC Press, 2008.

[26] Mikhasev, GI. On localized modes of free vibrations of single-walled carbon nanotubes embedded in nonhomogeneous elastic medium. ZAMM 2014; 94(1-2): 130-141.

[27] Mikhasev, GI, and Botogova, MG. Free localized vibrations of a long double-walled carbon nanotube introduced into an inhomogeneous elastic medium. Vestnik St. Petersburg University: Mathematics 2016; 49(1): 85-91.

[28] Tikhonov, AN, and Arsenin, VY. Solutions of ill-posed problems. Washington DC: Winston, 1977.

[29] Romano, G, Barretta, R, Diaco, M, et al. Constitutive boundary conditions and paradoxes in nonlocal elastic nanobeams. Int J Mech Sci 2017; 121: 151-156.

[30] Eringen, AC. Linear theory of nonlocal elasticity and dispersion of plane waves. Int J Eng Sci 1972; 10(5): 425-435.

[31] Eringen, AC. Theory of nonlocal elasticity and some applications. Res Mech 1987; 21: 313-342.

[32] Khodabakhshi, P, and Reddy, JN. A unified integro-differential nonlocal model. Int J Eng Sci 2015; 95: 60-75.

[33] Wang, YB, Zu, XW, and Dai, HH. Exact solutions for the static bending of Euler-Bernoulli beams using Eringen's two-phase local/nonlocal model. AIP Adv 2016; 6(8): 085114. 
[34] Challamel, N. Static and dynamic behaviour of nonlocal elastic bar using integral strain-based and peridynamic models. Comptes Rendus Mecanique 2018; 346: 320-335.

[35] Wang, YB, Huang, K, Lou, Z, et al. Exact solutions for the bending of Timoshenko beams using Eringen's two-phase nonlocal model. Math Mech Solids, to appear.

[36] Pisano, AA, and Fuschi, P. Closed form solution for a nonlocal elastic bar in tension. International Journal of Engineering Science 2003; 40(1): 13-23.

[37] Eptaimeros, KG, Koutsoumaris, CC, and Tsamasphyros, GJ. Nonlocal integral approach to the dynamical response of nanobeams. Int J Mech Sci 2016; 115: 68-80.

[38] Ari, N, and Eringen, AC. Nonlocal stress at Griffith crack. Cryst Latt Def Amorph Mat 1983; 10 : 33-38.

[39] Eringen, AC. Screw dislocation in nonlocal elasticity. J Phys D 1977; 10: 671-678.

[40] Eringen, AC. Edge dislocation in nonlocal elasticity. Int J Eng Sci 1977; 15: 177-183.

[41] Eringen, AC, and Balta, F. Edge dislocation in nonlocal hexagonal elastic crystal. Cryst Latt Def 1979; 8: 73-80.

[42] Lazar, M, and Agiasofitou, E. Screw dislocation in nonlocal anisotropic elasticity. Int J Eng Sci 2011; 49: 1404-1414.

[43] Liang, Y, and Han, Q. Prediction of nonlocal scale parameter for carbon nanotubes. Sci China Phys Mech 2012; 55 (9): 1670-1678.

[44] Liang, Y, Han, Q, and Xin, H. Elastic properties of carbon nanotubes. J Comput Theor Nanosci 2013; 10(5): 1061-1071.

[45] Gol'denveizer, AL, Theory of Thin Elastic Shells. International Series of Monograph in Aeronautics and Astronautics. New York: Pergamon Press, 1961. 